\section{Multidisciplinary management of periocular necrotising fasciitis: a series of 11 patients}

K Tambe' ${ }^{1}$ A Tripathi ${ }^{2}$ J Burns ${ }^{2}$ and R Sampath ${ }^{2}$

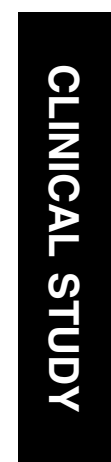

Introduction

Necrotising fasciitis is a rare and potentially fatal infection that spreads rapidly along the subcutaneous soft tissue planes. ${ }^{1}$ It is seen more commonly in immunocompromised or diabetic patients. The term was first used by Wilson in 1952 to describe the most consistent feature of the infection, necrosis of the fascia and subcutaneous tissue with relative sparing of the underlying muscle. ${ }^{2}$ It initially presents as cellulitis, and rapidly progresses along the superficial and deep facial planes leading to necrosis of the fascia and the overlying subcutaneous tissue and skin. Necrotising fasciitis can progress rapidly to systemic toxicity and even death if not promptly diagnosed and treated. To date, there have been sporadic case reports of periocular necrotising fasciitis and only a few small case series. ${ }^{3}$ We present a series of 11 patients with periocular necrotising fasciitis, which, to the best our knowledge, is the largest series in the literature. These patients were managed in our oculoplastic and orbit unit with a multidisciplinary team approach. The necessity of immediate resuscitation, early surgical debridement, and administration of broad spectrum intravenous antibiotics ${ }^{4}$ and the concurrent histopathological conformation of the diagnosis in the management of this potentially fatal condition is emphasised.

\section{Materials and methods}

This is a series of 11 patients diagnosed with necrotising fasciitis managed by our oculoplastic and orbit unit in conjunction with the plastic surgery, dermatology and microbiology teams. The patients presented over a period of 5 years, between January 2000 and December 2005 at a university teaching
${ }^{1}$ Lid, Lacrimal and Orbit Service, Department of Ophthalmology, Leicester Royal Infirmary, University Hospitals of Leicester, Leicester, UK

2Leicester Royal Infirmary, University Hospitals of Leicester, Leicester, UK

Correspondence: $\mathrm{K}$ Tambe, Lid, Lacrimal and Orbit Service, Department of Ophthalmology, Leicester Royal Infirmary, University Hospitals of Leicester, Infirmary Square, Leicester LE1 5WW, UK Tel: + 44 (0)116 258 6198; Fax: + 44 (0)116 2585927 E-mail: katya.tambe@ gmail.com

Received: 7 July 2010 Accepted in revised form: 15 August 2011 Published online: 9 December 2011 
hospital in the UK. The case notes, including documents presenting symptoms and signs, operative procedures, complications, and any further surgical rehabilitative surgery were analysed. Blood investigations, done at the time of initial presentation, and results of radiological imaging were also retrospectively reviewed and analysed, as were the reports of the histological examination of the debrided tissue.

\section{Results}

There were nine male and two female patients in our series. They ranged from 4-to- 84 years of age. Six of the patients had a history of preceding trauma; one patient developed necrotising fasciitis following chickenpox infection; and four of the patients had no significant initiating aetiology. The patients presented with varying degrees of unilateral or bilateral eyelid swelling, with or without facial and cervical involvement. All presented with rapidly progressing symptoms over $24-48 \mathrm{~h}$. Two patients were admitted directly to the high-dependency units because of fluctuating levels of consciousness. All patients had blood investigations carried out at the time of admission and a computed tomography scan (CT scan) of the affected region. They underwent urgent debridement of the necrotic skin and subcutaneous tissue, which was subjected to histopathological examination.

\section{Illustrative case report}

A Caucasian male, 68 years of age was admitted with a history of rapidly progressing left sided facial swelling associated with severe pain. This was preceded by a sixday history of a blister under his left nostril. The patient was known to suffer from Sweet syndrome (acute febrile neutrophilic dermatosis) for which he was immunosuppressed. On examination, there was swelling of the left side of the face involving both upper and lower eyelids, left cheek, left ear, left half of the neck, and upper chest (Figure 1). The skin was erythematous, warm to touch, and demonstrated serosanguinous blisters.

The eyelid skin showed necrotic patches and slough. Visual acuity was 6/9 in both eyes; there was no relative afferent papillary defect (RAPD); proptosis, or involvement of the extraocular muscles. Anterior and posterior segments were normal. The patient was febrile with a temperature of $38^{\circ} \mathrm{C}$. His blood investigations showed a haemoglobin of $6.7 \mathrm{~g} / \mathrm{dl}, \mathrm{WBC}$ (total white cell count $)=20.9$ cells $^{9} / 1$, CRP (C-reactive protein) $60 \mathrm{mg} / 1$, serum sodium $=139 \mathrm{mmol} / 1$, blood glucose $9.6 \mathrm{mmol} / 1$ and serum creatinine $=268 \mu \mathrm{mol} / 1$. Eyelid swabs for bacterial culture and sensitivity were taken. Urine analysis showed haematuria $(+++)$ and proteinuria $(+++)$. A diagnosis of necrotising fasciitis was made on the clinical appearance and history. The patient was immediately started on oral acyclovir, intravenous benzylpenicillin, flucloxacillin, and metronidazole, considering his past medical history of recurrent herpetic
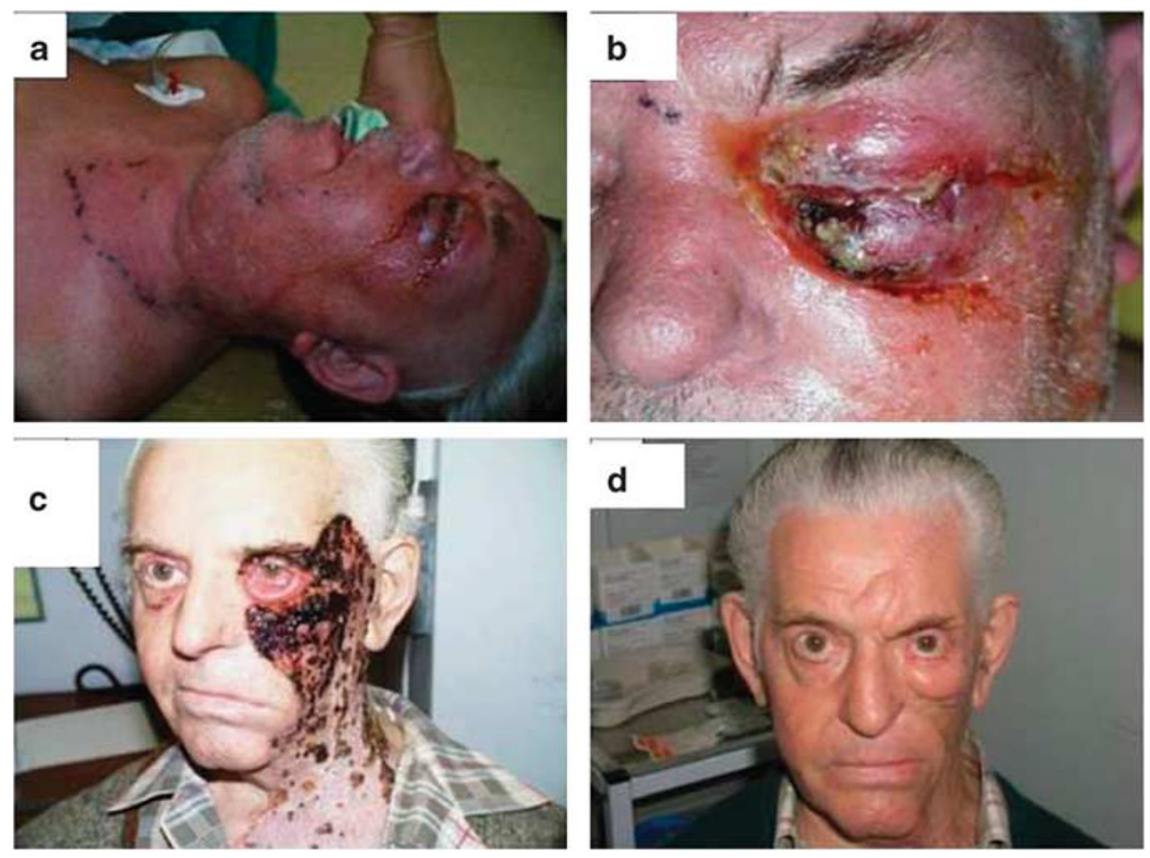

Figure 1 (a) Erythema and swelling of left side of face. (b) Necrosis of eyelid skin. (c) Cicatricial ectropion-sequelae of debridement. (d) Six months post reconstruction with forehead flap to the upper lid and full-thickness preauricular skin graft to the lower lid. 
infections and steroid therapy for Sweet Syndrome. The extent of skin involvement was marked, and urgent debridement of the necrotic tissue under a general anaesthetic was performed. Laryngeal oedema was noted on intubation and the patient required a tracheostomy. On histopathological examination, the debrided tissue showed acute inflammation and widespread areas of necrosis, affecting the skin, subcutaneous tissue, and fascia. An intraoperative swab taken for bacterial culture and sensitivity, grew Group A $\beta$-haemolytic Gram-positive streptococci. The diagnosis of necrotising fasciitis was confirmed by histopathology and culture from the necrotic wound and supported by the clinical presentation and blood results.

Postoperatively, the patient needed intensive care where he gradually recovered over three weeks.

Later, he developed a cictricial ectropion of the left lower eyelid and contracture of the left upper eyelid leading to corneal exposure, but with a good Bells phenomenon. He also developed severe contracture and scarring of the left side of his face and neck. Four months following the debridement, revision of scar tissue and reconstruction of the eyelids was performed with the help of a midline forehead flap to the upper lid and a full-thickness pre-auricular skin graft to the lower lid with an acceptable result. Following the reconstructive surgery, he maintained a good functional and cosmetic outcome. His vision and function were preserved in both eyes 3 years postoperatively.

\section{Results of the cohort of 11 patients}

All the patients presented with periorbital swelling and erythema with or with out necrosis of the overlying skin. All the patients were febrile on presentation with a temperature ranging from 37.9 to $38.3^{\circ} \mathrm{C}$. The blood pressure was stable in 10 of the 11 , and 1 patient was hypotensive on admission. Past medical history included Sweet syndrome with immunosuppression secondary to corticosteroids in one patient, and diabetes in four patients of which one patient had hyperglycaemia, acidosis, sepsis, and severe dehydration on admission. Blood investigation in the cohort of patients showed haemoglobin levels of 6.7 to $15.6 \mathrm{~g} / \mathrm{dl}$ (average $12.4 \mathrm{~g} / \mathrm{dl}$ ), total white blood cell count 7.1 to 29 cells $^{9} / 1$ (average 15.3 cells $^{9} / 1$ ), blood glucose level 4.8 to $11 \mathrm{mmol} / 1$ (average $9.38 \mathrm{mmol} / 1$ ), CRP 60 to $372 \mathrm{mg} / \mathrm{l}$ (average $191.75 \mathrm{mg} / \mathrm{l}$ ), serum sodium 129 to $140 \mathrm{mmol} / \mathrm{l}$ (average $137.62 \mathrm{mmol} / \mathrm{l}$ ), serum potassium 3.1 to $4.6 \mathrm{mmol} / 1$ (average $3.8 \mathrm{mmol} / \mathrm{l}$ ), serum urea 4.5 to $13.5 \mathrm{mg} / 1$ (average $8.01 \mathrm{mg} / \mathrm{l}$ ), serum creatinine 48 to $286 \mu \mathrm{mol} / 1$ (average $128.5 \mu \mathrm{mol} / 1$ ). All the patients were examined by a multidisciplinary team comprising oculoplastic surgeons, plastic surgeons, dermatologists, ENT surgeons, and miocrobiologists. All the 11 patients received broad-spectrum intravenous antibiotics, metronidazole and nasal oxygen. Urgent debridement of the nonviable tissue was expeditiously carried out in all the patients and the tissue was sent for histopathological examination. The histopathology showed acute and chronic inflammatory infiltration of the subcutaneous tissue and fascia, with widespread areas of necrosis of the overlying skin, as well as the subcutaneous tissue, itself consistent with necrotising fasciitis. Swabs were taken from the wound in all cases and sent for culture and sensitivity. All swabs grew gram-positive cocci of which eight were identified as Group A $\beta$-haemolytic streptococci; three patients also grew staphylococci. CT scan of the affected area demonstrated diffuse soft-tissue swelling. Of the 11 patients, 1 died because of systemic shock and multiorgan failure. Of the 10 surviving patients, 2 patients did not need any rehabilitative surgery and recovered well. One patient lost the sight in his affected eye secondary to a central retinal artery occlusion. The remaining eight patients required rehabilitative and reconstructive surgery, after the acute phase had settled. This was in the form of correction of lid malposition, ptosis correction, skin grafts, and flaps. Nine of the surviving ten patients had preservation of vision and good functional and cosmetic results.

\section{Discussion}

Necrotising fasciitis is an uncommon but life-threatening soft-tissue infection..$^{5-8}$ It is characterized by rapidly spreading inflammation and necrosis of skin, subcutaneous fat, and fascia and is more common in diabetics and immunosuppressed individuals. ${ }^{8,9}$ Although it can affect any age group, adults are known to be more commonly affected than children. ${ }^{6}$ A review of 163 patients with necrotising fasciitis showed that $10 \%$ involve the head and neck region. ${ }^{10}$ Necrotising fasciitis rarely involves the periocular skin, with less than 60 well-documented case reports over the last 50 years. ${ }^{11-13}$ Clinically, there are three subtypes of necrotising fasciitis, namely fulminant, acute, and sub-acute. The fulminant variety is characterised by a very rapid onset and progression and, in severe cases, can result in circulatory shock and multi-organ failure. The acute variety progresses over a few days and generally involves large areas of skin. The subacute type progresses insidiously over a number of weeks and only a localised area may be affected. ${ }^{8} \beta$-Haemolytic streptococci are responsible for fulminant and acute cases, whereas sub-acute cases are generally polymicrobial. ${ }^{6,8,14,15}$ The incidence of necrotising fasciitis has increased in the past decade as a result of the resurgence of group A streptococcal infection. ${ }^{16}$ The streptococcal $M$ protein has been identified as a potent inducer of inflammation and seems 
to be responsible for the severity of necrotising fasciitis. ${ }^{17}$ Other bacteria have also been implicated in the pathogenesis of necrotising fasciitis like Escherichia coli, ${ }^{18}$ Kleibsella pneumoniae, ${ }^{19}$ and Moraxella. ${ }^{20}$

Trauma to the eyelid in the form of minor blunt injury, insect bites, surgical blepharoplasty, ${ }^{21,22}$ and laser blepharoplasty ${ }^{23}$ have been reported as causative factors in the development of necrotising fasciitis. But, it may develop de novo in nontraumatised skin as well. Thrombosis of the microcirculatory vessels of superficial fascia leads to secondary gangrene of the skin and forms the basis for the pathogenesis of this condition. Periocular necrotising fasciitis can have devastating complications. Central retinal artery occlusion with loss of vision and exenteration for deep orbital involvement, has been reported. ${ }^{24}$ Early surgical intervention reduces morbidity and mortality significantly; left untreated, the mortality ranges between $30-70 \% .^{7,23}$ In patients with periorbital necrotising fasciitis, the mortality rate has been reported to be $10 \% .^{12,15}$

Diagnosis is difficult in the early course of the disease because of the paucity of cutaneous findings. ${ }^{8,25}$ Also, the use of pre-admission antibiotics modify the clinical picture adding to the diagnostic difficulty. ${ }^{8}$ LRINEC (Laboratory risk indicator for diagnosis of necrotising fasciitis) is considered a robust way to detect early cases and involves scoring blood investigations including the total white blood count (WBC), C-reactive protein (CRP), haemoglobin level, serum sodium level, serum creatinine level, and the blood glucose level. ${ }^{26}$ This clinical score was developed on the basis of retrospective studies, and has not been validated prospectively. Our series of cases had a score ranging from 0 to 8,4 patients had a score of 6 or more and 1 patient had a score of 0 . The scores did not correlate with the severity of the condition. It would be useful in future studies on periocular necrotising fasciitis to be able to identify an alternative indicator that is more sensitive for the early detection of necrotising fasciitis than the LRINEC score. Imaging in the form of X-rays, CT Scans, and MRI have been used to help in the diagnosis, but these modalities may not demonstrate facial involvement early in the disease process. ${ }^{24,27,28}$

The mode of presentation is with pale-red, tense, swollen skin. The patient complains of severe pain and is febrile. The infection spreads subcutaneously, and, within 1 to 2 days, the skin becomes cyanotic with irregular erythematous borders and serosanguinous bullae. Secondary gangrene of the overlying fascia and skin develops within hours to 4 to 5 days. ${ }^{29}$ The necrotising fasciitis may involve both the eyelids owing to the minimal resistance offered by the subcutaneous tissue over the bridge of the nose. ${ }^{29}$

The key to successful management of necrotising fasciitis is early diagnosis and intervention. ${ }^{6,14,19}$ The use of hyperbaric oxygen and intravenous immunoglobulin have been recommended by some researchers, but are more useful as adjuncts to broad-spectrum intravenous antibiotic therapy and early debridement. ${ }^{30-32}$ It is vital for the patient to understand the seriousness of the condition, the implications if urgent surgical debridement is not undertaken, and the consequences and sequelae of the surgical debridement itself. In our series of 11 patients, 1 patient died of severe systemic complications, and 1 patient lost the sight secondary to a central retinal artery occlusion. Yet, prompt management, in the form of intravenous antibiotics and surgical debridement, saved not only the vision but also the lives of the other nine patients. Eight patients required rehabilitative and reconstructive surgery to the eyelids giving a good cosmetic and functional outcome.

To conclude, necrotising fasciitis is a serious and potentially disfiguring and life-threatening infection. As it can occur after routine procedures like blepharoplasty and is potentially blinding, it is important for ophthalmologists to be able to diagnose and manage this condition. A high index of suspicion is needed for diagnosis that is predominantly clinical, and for which biochemical, and imaging are useful adjuncts.

Histopathology is diagnostic. CT Scan of the affected area did not modify the management of the patients in our series. The LRINEC score, calculated in retrospect for each patient, did not correlate with the severity of the necrotising fasciitis in our series. A multidisciplinary approach for the comprehensive care of these patients is mandatory. While managing these patients, intensive care facilities should be made available and long-term follow-up and management of complications must be discussed and planned with the patient.

\section{Summary}

What was known before

- Necrotising fasciitis is a serious and potentially disfiguring and life-threatening infection. As it can occur after routine procedures like blepharoplasty and is potentially blinding, it is important for ophthalmologists to be able to diagnose and manage this condition. There are a handful of case series in the literature on this condition; the most recent is a series of seven patients in Ophthalmology.

What this study adds

- This article reviews the previous literature and emphasizes that a high index of suspicion is needed for diagnosis that is clinical and biochemical. The LRINEC criteria, a more recently developed diagnostic tool for necrotising fasciitis, which was applied retrospectively to our group of patients, was not found to correlate with the severity of periocular necrotising fasciitis. A multidisciplinary approach for the comprehensive care is mandatory. While managing these patients, intensive care facilities should be made available and long-term follow-up and management of complications must be discussed and planned with the patient. 


\section{Conflict of interest}

The authors declare no conflict of interest.

\section{References}

1 Bisno AL, Stevens DL. Streptococcal infections of the skin and soft tissues. N Engl J Med 1996; 334: 240-245.

2 Wilson B. Necrotising fasciitis. Am Surg 1952; 18: 416-431.

3 Shayegani A, MacFarlane D, Kazim M, Grossman ME. Streptococcal gangrene of the eyelids and orbit. Am J Ophthalmol 1995; 120: 784-792.

4 Hasham S, Matteucci P, Stanley PRW, Hart NB. Necrotising fasciitis. Br Med J 2005; 330: 830-833.

5 Sehgal VN, Sehgal N, Sehgal R, Khandpur S, Sharma S. Necrotising fasciitis. J Dermatolog Treat 2006; 17(3): 184-186.

6 Legbo JN, Shehu BB. Necrotizing fasciitis: a comparative analysis of 56 cases. J Natl Med Assoc 2005; 97(12): 1692-1697.

7 Young MH, Aronoff DM, Engleberg NC. Necrotizing fasciitis: pathogenesis and treatment. Expert Rev Anti Infect Ther 2005; 3(2): 279-294.

8 Wong CH, Chang HC, Pasupathy S, Khin LW, Tan JL, Low CO. Necrotizing fasciitis: clinical presentation, microbiology, and determinants of mortality. J Bone Joint Surg Am 2003; 85-A(8): 1454-1460.

9 Vinh DC, Embli JM. Severe skin and soft tissue infections and associated critical illness. Curr Infect Dis Rep 2006; 8(5): 375-383.

10 Childers BJ, Potyondy LD, Nachreiner R, Rogers FR, Childers ER, Oberg KC et al Necrotising fasciitis: a fourteen-year retrospective study of 163 consecutive patients. Am Surg 2002; 68: 109-116.

11 Kronish JW, McLeish WM. Eyelid necrosis and periorbital necrotizing fasciitis: report of a case and review of literature. Ophthalmology 1991; 98: 92-98.

12 Walters R. A fatal case of necrotizing fasciitis of the eyelid. Br J Ophthalmol 1988; 72: 428-431.

13 Rose GE, Howard DJ, Watts MR. Periorbital necrotizing fasciitis. Eye 1991; 5: 736-740.

14 Marshall DH, Jordan DR, Gilberg SM, Harvey J, Arthurs BP, Nerad J. Periocular necrotizing fasciitis: a review of five cases. Ophthalmology 1997; 104(11): 1857-1862.

15 Gauzit R. Necrotizing skin and soft tissue infections: definitions, clinical and microbiological features. Ann Fr Anesth Reanim 2006; 25(9): 960-970.

16 Low DE, McGeer A. Skin and soft tissue infection: necrotizing fasciitis. Curr Opin Infect Dis 1998; 11(2): 119-123.

17 Pahlman LI, Morgelin M, Eckert J, Johansson L, Russell W, Riesbeck K et al. Streptococcal M protein: a multipotent and powerful inducer of inflammation. J Immunol 2006; 177(2): 1221-1228.

18 Li DM, Lun LD, Chen XR. Necrotising fasciitis with Escherichia coli. Lancet Infect Dis 2006; 6(7): 456.

19 Liu YM, Chi CY, Ho MW, Chen CM, Liao WC, Ho CM et al. Microbiology and factots affecting mortality in necrotizing fasciitis. J Microbiol Immunol Infect 2005; 38(6): 430-435.

20 Brittain CJ, Penwarden A, Mearza A, Verity D. Moraxella as a cause of necrotizing fasciitis of the eyelid. Eye 2006; 20(11): 1312-1314.

21 Suner IJ, Meldrum ML, Johnson TE, Tse DT. Necrotizing fasciitis after cosmetic blepharoplasty. Am J OPhthalmol 1999; 128(3): 367-368.

22 Ray AM, Bressler K, Davis RE, Gallo JF, Patete ML. Cervicofacial necrotizing fasciitis. A devastating complication of blepharoplasty. Arch Otolaryngol Head Neck Surg 1997; 123(6): 633-636.

23 Jordan DR, Mawn L, Marshall DH. Necrotizing fasciitis caused by group A streptococcus infection after laser blepharoplasty. Am J Ophthalmol 1998; 125(2): 265-266.

24 Elner VM, Demirci H, Nerad JA, Hassan AS. Periocular necrotizing fasciitis with visual loss, pathogenesis and treatment. Ophthalmology 2006; 113: 2338-2345.

25 Wong $\mathrm{CH}$, Wang YS. The diagnosis of necrotizing fasciitis. Curr Opin Infect Dis 2005; 18(2): 101-106.

26 Wong $\mathrm{CH}$. The LRINEC (Laboratory Risk Indicator for Necrotizing Fasciitis) score: a tool for distinguishing necrotizing fasciitis from other soft tissue infections. Crit Care Med 2004; 32: 1535-1541.

27 Schmid MR, Kossmann T, Duewell S. Differentiation of necrotising fasciitis and cellulitis using MR imaging. Am J Roentgenol 1998; 170: 615-620.

28 Arslan A, Pierre-Jerome C, Borthne A. Necrotising fasciitis: unreliable MRI findings in the preoperative diagnosis. Eur J Radiol 2000; 36: 139-143.

29 Wyoski MG, Santora TA, Shah RM, Friedman AC. Necrotising fasciitis: CT characteristics. Radiology 1997; 203: 859-863.

30 Escobar SJ, Slade Jr JB, Hunt TK, Cianci P. Adjuvant hyperbaric oxygen therapy (HBO2) for treatment of necrotizing fasciitis reduces mortality and amputation rate. Undersea Hyperb Med 2005; 32(6): 437-443.

31 Lazzeri D, Lazzeri S, Figus M, Agostini T, Nardi M, Pantaloni M. Hyperbaric oxygen therapy as further adjunctive therapy in the treatment of periorbital necrotizing fasciitis caused by group A Streptococcus. Ophthal Palst Reconstr Surg 2010; 26(6): 504-505.

32 Aakalu VK, Sajja K, Cook JL, Ahmad AZ. Group A Streptococcal necrotizing fasciitis of the eyelids and face managed with debridement and adjunctive intravenous immunoglobulin. OPhthal Plast Reconstr Surg 2009; 25 : 332-334. 